\title{
Local and Systemic Cardiovascular Effects from Monochromatic Infrared Therapy in Patients with Knee Osteoarthritis: A Double-Blind, Randomized, Placebo-Controlled Study
}

\author{
Ru-Lan Hsieh, ${ }^{1,2,3}$ Wei-Cheng Liao, ${ }^{2}$ and Wen-Chung Lee ${ }^{4}$ \\ ${ }^{1}$ Department of Physical Medicine and Rehabilitation, Shin Kong Wu Ho-Su Memorial Hospital, 95 Wen-Chang Road, \\ Shih-Lin District, Taipei 111, Taiwan \\ ${ }^{2}$ School of Medicine, College of Medicine, Taipei Medical University, 250 Wuxing Street, Taipei 110, Taiwan \\ ${ }^{3}$ School of Nursing and Management in Gerontology, College of Nursing, Taipei Medical University, 250 Wuxing Street, \\ Taipei 110, Taiwan \\ ${ }^{4}$ Institute of Epidemiology and Preventive Medicine, College of Public Health, National Taiwan University, 17 Xuzhou Road, \\ Taipei 100, Taiwan
}

Correspondence should be addressed to Ru-Lan Hsieh, m001052@ms.skh.org.tw

Received 10 February 2012; Revised 27 April 2012; Accepted 27 April 2012

Academic Editor: Gerhard Litscher

Copyright (c) 2012 Ru-Lan Hsieh et al. This is an open access article distributed under the Creative Commons Attribution License, which permits unrestricted use, distribution, and reproduction in any medium, provided the original work is properly cited.

\begin{abstract}
Infrared (IR) therapy is used for pain relief in patients with knee osteoarthritis (OA). However, IR's effects on the cardiovascular system remain uncertain. Therefore, we investigated the local and systemic cardiovascular effects of monochromatic IR therapy on patients with knee OA in a double-blind, randomized, placebo-controlled study. Seventy-one subjects with knee OA received one session of $40 \mathrm{~min}$ of active or placebo monochromatic IR treatment (with power output of $6.24 \mathrm{~W}$, wavelength of $890 \mathrm{~nm}$, power density of $34.7 \mathrm{~mW} / \mathrm{cm}^{2}$ for $40 \mathrm{~min}$, total energy of $41.6 \mathrm{~J} / \mathrm{cm}^{2}$ per knee per session) over the knee joints. Heart rate, blood pressure, and knee arterial blood flow velocity were periodically assessed at the baseline, during, and after treatment. Data were analyzed by repeated-measure analysis of covariance. Compared to baseline, there were no statistically significant group $\mathrm{x}$ time interaction effects between the 2 groups for heart rate $(P=0.160)$, blood pressure (systolic blood pressure: $P=0.861$; diastolic blood pressure: $P=0.757)$, or mean arterial blood flow velocity $(P=0.769)$ in follow-up assessments. The present study revealed that although there was no increase of knee arterial blood flow velocity, monochromatic IR therapy produced no detrimental systemic cardiovascular effects.
\end{abstract}

\section{Introduction}

Osteoarthritis (OA) generally involves articular cartilage, anabolic and catabolic mechanisms, and bony structures in the synovial joints [1]. Weaker quadriceps muscle strength, lower knee proprioception, and poor balance with increased postural swaying were noted in subjects with knee OA than in age- and gender-matched controls [2]. Pain and decreased postural stability may be accompanied by difficulties in performing basic and instrumental daily activities, increased fall risks among community-dwelling elderly $[3,4]$, and a decreased quality of life [5].

Physical modalities are commonly used to treat older patients with knee $\mathrm{OA}$ to ameliorate pain and improve functional performance in the rehabilitation medical field. Physical modalities, such as hot packs, pulse ultrasound, transcutaneous electrical nerve stimulation, and phototherapy, are commonly applied to patients with musculoskeletal pain to increase local circulation [6]. However, there are few high-quality clinical studies with randomized placebocontrolled designs on physical modalities' therapeutic effects in the rehabilitation medicine field [7].

Light encompasses a portion of the electromagnetic spectrum. Infrared (IR) radiation wavelengths range from $750 \mathrm{~nm}$ to $1 \mathrm{~mm}$. In 2002, the US Food and Drug Administration approved IR therapy for pain relief associated with neck and head pain, arthritis, and carpal tunnel syndrome [8]. IR therapy is commonly used for patients with wounds, 
lower-limb peripheral neuropathies, and musculoskeletal disorders such as knee OA [7, 9-19]. Photoenergy exerts bioenergetic, biostimulating, biochemical, and bioelectrical effects on cells $[20,21]$. The biological effect of phototherapy is related to photochemical cellular reactions rather than thermal reactions [22]. Phototherapy has been found to improve microcirculation by increasing arterioles diameter and blood flow velocity [23-25]. Improving microcirculation at the local and systemic levels is one of the most important phototherapy effects $[26,27]$. It is speculated that vessel dilatation, increase of blood flow rate, and improved blood rheologic properties are mediated by NO, prostacyclin, and endothelial-derived hyperpolarizing factors, all of which are produced by endothelial cells $[26,28]$. NO causes rapid transduction and increases local blood flow followed by prostacyclin and endothelial-derived hyperpolarizing factors in changing microcirculation at the systemic level [26, 29]. In addition to mediation by enhancing $\mathrm{NO}$ synthesis and increasing microcirculation, phototherapy also relives pain by other pathways and mechanisms, such as by modulating inhibitory cyclooxygenase and prostaglandin E2, modulating nerve transmission, increasing endorphin and serotonin release, and stimulating metabolism $[8,21,30]$.

A series of IR treatments had been confirmed to have significant efficacy in improving pain, function, and quality of life in patients with knee OA [17, 31, 32]. Possible mechanisms include peripheral nerve stimulation, microcirculation enhancement, analgesic effects, inflammation resolution, chondrocyte proliferation enhancement, and increased matrix synthesis $[17,33]$. Due to significant efficacy of OA knee treatment with IR therapy as a series of sessions, it is necessary to provide evidence that IR therapy does not produce any detrimental systemic cardiovascular effect. However, to our knowledge, no comprehensive study has focused on IR therapy's cardiovascular effects in patients with knee OA [32, 34, 35].

If IR therapy in patients with knee OA can improve the knee arterial blood flow velocity without producing detrimental systemic cardiovascular effects, then the increased blood flow in and/or around the knee joint may infer benefits to the knee joint such as pain reduction in patients with knee OA by long term, repeated IR treatments. Therefore, we hypothesized that IR therapy presumably would influence knee joint tissue perfusion by increasing the local arterial blood velocity at the knee without producing detrimental systemic cardiovascular effects. In our research, we conducted a double-blind, randomized, and placebo-controlled study to examine local and systemic cardiovascular effects from monochromatic IR therapy in patients with knee OA.

\section{Materials and Methods}

This study was conducted at Shin Kong Wu Ho-Su Memorial Hospital, a teaching hospital with 921 beds located in northern Taiwan. In total, 73 subjects confirmed to have knee OA were identified and recruited from the clinic of the Department of Physical Medicine and Rehabilitation at the hospital. All patients fulfilled the combined knee OA clinical and radiographic criteria established by the American
College of Rheumatology [36]. Anteroposterior radiographic views of both knees were taken while bearing weight. A qualified senior physiatrist was in charge of reading the $\mathrm{X}$-rays to classify subjects' Kellgren-Lawrence scores. The hospital's Institutional Review Board for the Protection of Human Subjects approved this study. Written informed consent was obtained from each subject. Subjects with a history of stroke, peripheral vascular disease, peripheral neuropathy, a previous knee operation with an implant, a malignancy, or who were pregnant or planning to become pregnant were excluded.

General information, including age, gender, educational level, marital status, work status, smoking and drinking habits, and comorbidities, was recorded. The body mass index (BMI) was calculated. The self-reported OA kneespecific health status was assessed with the Chinese version of the Western Ontario and McMaster University Osteoarthritis Index (WOMAC) [37]. Using a visual analog scale, total WOMAC scores of pain, stiffness, and physical function, respectively vary from 0 to 500,0 to 200 , and 0 to 1700 . Higher scores represent worse symptoms with greater functional limitation. The reliability and validity of the three visual analog scale versions are excellent [37-40].

After completing basic data recording, patients were allocated to a treatment group (active treatment) or a placebo group (inactive treatment) following the block randomization principle (with a block size of four). The allocation was initially concealed. An envelope was opened for each consecutive subject to reveal his or her group assignment at the time when he or she was recruited to the study. All patients, regardless of group assignment, underwent $40 \mathrm{~min}$ of monochromatic IR therapy with either power on (treatment group) or power off (placebo group) (Figure 1).

Each subject laid down on a standard bed with socks, shoes, and pants removed and rested for $15 \mathrm{~min}$ before the intervention in a quiet room with air conditioning. An Anodyne Therapy Professional System (Anodyne Therapy Professional System 480) was used in this study. The device has a main power unit with 8 flexible therapy pads. Each pad contains 60 superluminous gallium-aluminum arsenide diodes that emit an $890 \mathrm{~nm}$ light energy wavelength. Eight therapeutic pads were used in this study for both knees, and subjects in the treatment group received a total energy of $41.6 \mathrm{~J} / \mathrm{cm}^{2}$ per knee per session (with a radiant power output of $6.24 \mathrm{~W}$, at a wavelength of $890 \mathrm{~nm}$, and a power density of $34.7 \mathrm{~mW} / \mathrm{cm}^{2}$ for $40 \mathrm{~min}$ ).

Four therapy pads were placed over the following sites in each knee: the anterior knee joint, the posterior knee joint, and the medial and lateral knee joints (Figure 2). The pads were held in place with neoprene straps supplied by the manufacturer. All subjects were told that they may or may not feel anything from the treatment. Subjects received 1 session of monochromatic IR therapy for $40 \mathrm{~min}$ with either the power on or off. The manufacturer checked the monochromatic IR device before the intervention began on the first participant.

The heart rate and blood pressure were measured over the brachial artery with an automated sphygmomanometer (Tango $^{+}$Stress BP, Sun Tech Medical Instruments) by 


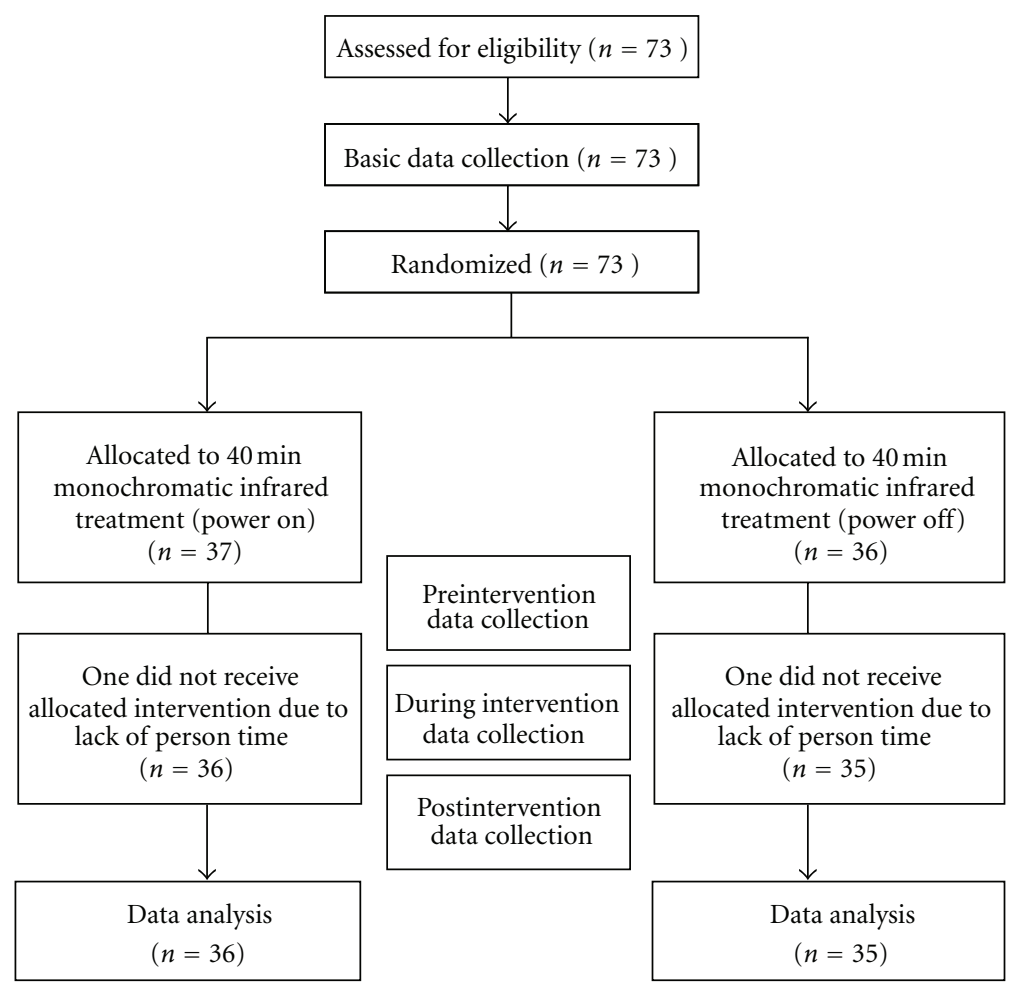

Figure 1: Consort flow diagram.

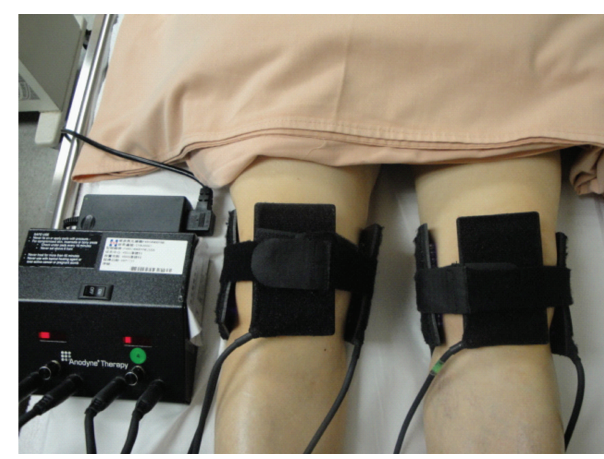

FIGURE 2: Monochromatic infrared therapy application.

a well-trained technician. The blood pressure measurement had good reliability and validity [41-43]. The instrument was calibrated, and the same cuff was used for all subjects. The heart rate was monitored before treatment, immediately after completing $40 \mathrm{~min}$ of treatment, and 5, 10, and $15 \mathrm{~min}$ after completing treatment. Systolic and diastolic blood pressures were automatically recorded before monochromatic IR treatment, every $10 \mathrm{~min}$ during the $40 \mathrm{~min}$ of treatment, and 5 and 15 min after completing treatment. No conversation was allowed between the participants and the technician during the whole course of heart rate and blood pressure measurements.

Color Doppler ultrasonography (LOGIQ P5, GE Ultrasound Korea, General Electric) was performed on patients in a prone position by a qualified senior physiatrist who was not informed of each patient's group allocation. The peak popliteal arterial systolic blood flow velocity (meters/second) was measured in each subject by high-resolution B-mode ultrasound images using standardized parameters with a 7.5 MHz linear array transducer. The peak popliteal arterial blood flow velocity was measured before IR radiation treatment; immediately after completion of 40 min of treatment; and 5, 10, and 15 minutes after treatment. It has high reliability $[44,45]$.

Except for the physical therapist performing the monochromatic IR treatments, neither the subjects receiving the treatment nor the investigators (including the technician who measured patients' heart rates and blood pressures, and the physiatrist who conducted the Doppler study) were aware of the monochromatic IR therapy's operating status during the study's treatment and data collection periods.

The results are expressed as the mean \pm the standard deviation. A chi-squared test or $t$-test was used to analyze demographic data such as age, gender, educational level, marital status, occupation, comorbidities, smoking and drinking habits, BMI, Kellgren-Lawrence scores, and knee OA-specific measures of pain, stiffness, and physical function of the treatment and placebo groups. Repeated-measure analysis of covariance (ANCOVA) was used to assess the heart rate, systolic and diastolic blood pressures, and mean arterial knee joint blood flow in patients with knee OA between the follow-up assessments in each group, using the pretreatment baseline as the covariate. The group effect, time effect, and group $\mathrm{x}$ time interaction effect for the 2 groups at the follow-up assessments were analyzed. We report the 
TABle 1: Patient's basic demographics.

\begin{tabular}{|c|c|c|c|}
\hline \multirow[b]{2}{*}{ Variables } & \multicolumn{2}{|c|}{ Groups } & \multirow[b]{2}{*}{$P$ value } \\
\hline & $\begin{array}{c}\text { Treatment } \\
n=36\end{array}$ & $\begin{array}{c}\text { Placebo } \\
n=35\end{array}$ & \\
\hline \multicolumn{4}{|l|}{ Gender } \\
\hline Female & $33(92 \%)$ & $28(80 \%)$ & 0.189 \\
\hline Male & $3(8 \%)$ & $7(20 \%)$ & \\
\hline Age (yr) & $61.1 \pm 9.3$ & $61.3 \pm 12.0$ & 0.931 \\
\hline Body mass index $\left(\mathrm{kg} / \mathrm{m}^{2}\right)$ & $26.4 \pm 5.0$ & $26.0 \pm 4.5$ & 0.765 \\
\hline \multicolumn{4}{|l|}{ Married } \\
\hline Yes & $28(78 \%)$ & $26(74 \%)$ & 0.730 \\
\hline \multicolumn{4}{|l|}{ Educational level } \\
\hline Below 9th grade & $21(58 \%)$ & $19(53 \%)$ & 0.650 \\
\hline Above 9th grade & $15(42 \%)$ & $16(47 \%)$ & \\
\hline \multicolumn{4}{|l|}{ Work status } \\
\hline Yes & $6(17 \%)$ & $4(12 \%)$ & 0.735 \\
\hline \multicolumn{4}{|l|}{ Comorbidities } \\
\hline Yes & $18(50 \%)$ & $21(62 \%)$ & 0.322 \\
\hline \multicolumn{4}{|l|}{ Smoking } \\
\hline Yes & $0(0 \%)$ & $0(0 \%)$ & 1.000 \\
\hline \multicolumn{4}{|l|}{ Alcohol consumption } \\
\hline Yes & $3(8 \%)$ & $3(9 \%)$ & 1.000 \\
\hline Kellgren-Lawrence scores & $2.7 \pm 0.7$ & $2.7 \pm 0.7$ & 0.962 \\
\hline \multicolumn{4}{|l|}{ WOMAC* } \\
\hline Pain & $130.0 \pm 87.9$ & $116.9 \pm 84.4$ & 0.493 \\
\hline Stiffness & $40.4 \pm 47.2$ & $40.6 \pm 40.6$ & 0.986 \\
\hline Physical function & $413.3 \pm 318.1$ & $413.5 \pm 326.8$ & 0.999 \\
\hline
\end{tabular}

Note: the scores are presented as the number of cases (percentage) or the mean (standard deviation) for each variable.

*WOMAC: Western Ontario and McMaster University Osteoarthritis Index.

results of the ANCOVA by providing the $F$ statistic, degrees of freedom, and the $P$ value for all 71 participants. The level of statistical significance was set at $P<0.05$.

\section{Results}

Seventy-three subjects were enrolled in this study. Two subjects refused to participate after completing basic data collection due to personal time constraints. There was no statistically significant difference in the 2 groups in age, gender, educational level, marital status, occupation, comorbidities, smoking and drinking habits, BMI, or severity of knee OA according to the Kellgren-Lawrence scores and WOMAC assessments. Detailed demographic data for both groups are shown in Table 1.

Compared to pretreatment, there was no statistical significance demonstrated in the heart rate between the 2 groups (group effect: $P=0.918$; time effect: $P=0.340$; group $\mathrm{x}$ time interaction effect: $P=0.160$ ) during the 4 follow-up assessments (after 40 min of treatment; and 5, 10, and 15 min after treatment) (Table 2, Figure 3).

As shown in Table 2 and Figure 4, there was no statistically significant difference in systolic blood pressure (group effect: $P=0.281$; time effect: $P=0.180$; group $\mathrm{x}$ time

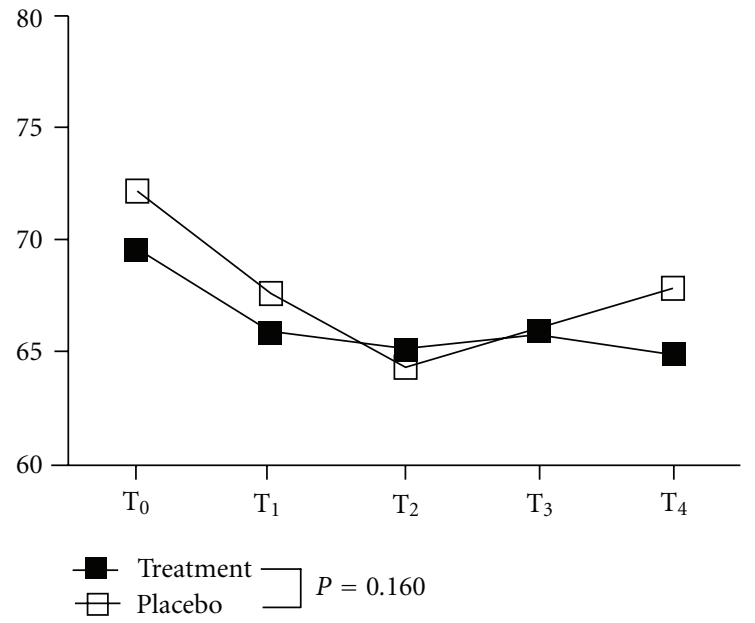

FIGURE 3: Changes in the heart rate with monochromatic infrared treatment. Solid square: treatment group; hollow square: placebo group; $\mathrm{T}_{0}$ : before treatment; $\mathrm{T}_{1}$ : after $40 \mathrm{~min}$ of treatment; $\mathrm{T}_{2}$ : 5 min after treatment; $\mathrm{T}_{3}: 10$ min after treatment; $\mathrm{T}_{4}: 15$ min after treatment. Between groups by repeated-measure ANCOVA: group effect: $P=0.918\left(F_{1,68}=0.01\right)$; time effect: $P=0.340\left(F_{3,204}=1.12\right)$; group x time interaction effect: $P=0.160\left(F_{3,204}=1.74\right)$.

interaction effect: $P=0.861)$ or diastolic blood pressure (group effect: $P=0.262$; time effect: $P=0.663$; or group $\mathrm{x}$ time interaction effect: $P=0.757$ ) between the 2 groups during the 6 follow-up assessments (after 10, 20, 30, and $40 \mathrm{~min}$ of the treatment; and 5 and $10 \mathrm{~min}$ after the treatment was completed).

As for the popliteal arterial blood flow velocity, compared to pretreatment, there was no statistically significant difference in the blood flow (group effect: $P=0.666$, time effect: $P=0.323$, group $\mathrm{x}$ time interaction effect: $P=0.769)$ at the 4 separate follow-up assessments between the 2 groups (after 40 min of treatment; and 5, 10, and 15 min after treatment) (Table 2, Figure 5).

No local or systemic side effects were reported during or after the intervention.

\section{Discussion}

A series of IR treatments are demonstrated to have significant efficacy in improving pain in patients with knee OA. However, the cardiovascular effects by these treatments remain uncertain. To our knowledge, this is the first study to demonstrate the local and systemic cardiovascular effects of monochromatic IR therapy in patients with knee OA. Our results revealed that although there was no knee arterial blood flow velocity increase, monochromatic IR therapy produced no detrimental systemic cardiovascular effects.

A significant microcirculation increase began after $20 \mathrm{~min}$ of IR therapy and reached a maximal level $15 \mathrm{~min}$ after treatment termination [46]. Therefore, we conducted 40 min of monochromatic IR therapy and followed up for 15 min after treatment termination to examine the local and systemic cardiovascular effects in patients with knee OA in the present study. There has been a tendency to shift from 
TABLE 2: Changes in heart rate, blood pressure, and blood flow velocity.

\begin{tabular}{|c|c|c|c|c|c|c|}
\hline \multirow[t]{2}{*}{ Time point } & \multirow{2}{*}{$\begin{array}{l}\text { Treatment } \\
\text { group } \\
\text { Mean (SD) }\end{array}$} & \multirow{2}{*}{$\begin{array}{l}\begin{array}{l}\text { Placebo } \\
\text { group }\end{array} \\
\text { Mean (SD) }\end{array}$} & \multirow{2}{*}{$\begin{array}{l}\text { Mean between } \\
\text { group difference } \\
\text { (95\% confidence } \\
\text { interval) }\end{array}$} & \multicolumn{3}{|c|}{$\begin{array}{l}\text { Repeated-measure ANOVA } \\
\quad P \text { value }(F \text { value })\end{array}$} \\
\hline & & & & Group & Time & Time $\times$ group \\
\hline \multicolumn{7}{|l|}{ Heart rate (beats/min) } \\
\hline Initial score & $69.6(10.2)$ & $72.2(11.5)$ & $-2.3(-7.4,2.8)$ & 0.918 & 0.340 & 0.160 \\
\hline After 40 min of treatment & $65.8(8.0)$ & $67.6(11.2)$ & $-1.8(-6.4,2.8)$ & $\left(F_{1,68}=0.01\right)$ & $\left(F_{3,204}=1.12\right)$ & $\left(F_{3,204}=1.74\right)$ \\
\hline 5 min after treatment & $65.1(8.5)$ & $64.3(10.4)$ & $-0.2(-4.8,4.5)$ & & & \\
\hline 10 min after treatment & $65.8(9.2)$ & $65.8(10.7)$ & $-0.9(-5.9,4.1)$ & & & \\
\hline 15 min after treatment & $64.9(10.1)$ & $67.8(11.7)$ & $-3.6(-8.7,1.6)$ & & & \\
\hline \multicolumn{7}{|l|}{ Systolic blood pressure (mmHg) } \\
\hline Initial score & $120.3(16.5)$ & $121.1(17.6)$ & $-0.8(-8.9,7.3)$ & 0.281 & 0.180 & 0.861 \\
\hline After 10 min of treatment & $111.8(17.0)$ & $114.0(20.7)$ & $-2.2(-11.1,6.8)$ & $\left(F_{1,68}=1.18\right)$ & $\left(F_{5,335}=1.53\right)$ & $\left(F_{5,335}=0.38\right)$ \\
\hline After 20 min of treatment & $109.3(17.3)$ & $114.6(24.8)$ & $-5.3(-15.4,4.8)$ & & & \\
\hline After 30 min of treatment & $111.6(17.5)$ & $117.3(21.0)$ & $-5.7(-14.8,3.5)$ & & & \\
\hline After 40 min of treatment & $112.8(20.4)$ & $116.4(14.5)$ & $-3.6(-12.0,4.8)$ & & & \\
\hline 5 min after treatment & $116.1(16.8)$ & $117.4(19.6)$ & $-1.3(-10.0,7.3)$ & & & \\
\hline 15 min after treatment & $114.1(15.0)$ & $119.9(17.3)$ & $-5.9(-13.6,1.8)$ & & & \\
\hline \multicolumn{7}{|c|}{ Diastolic blood pressure (mmHg) } \\
\hline Initial score & $73.8(12.0)$ & $70.8(11.3)$ & $3.0(-2.5,8.5)$ & 0.262 & 0.663 & 0.757 \\
\hline After $10 \mathrm{~min}$ of treatment & $73.1(12.0)$ & $70.5(12.0)$ & $2.6(-3.1,8.3)$ & $\left(F_{1,68}=1.28\right)$ & $\left(F_{5,335}=0.65\right)$ & $\left(F_{5,335}=0.53\right)$ \\
\hline After 20 min of treatment & $69.7(12.7)$ & $70.1(12.7)$ & $-0.4(-6.5,5.6)$ & & & \\
\hline After $30 \mathrm{~min}$ of treatment & $70.6(10.8)$ & $72.9(14.5)$ & $-2.3(-8.4,3.7)$ & & & \\
\hline After 40 min of treatment & $72.3(14.1)$ & $72.8(11.8)$ & $-0.5(-6.6,5.7)$ & & & \\
\hline 5 min after treatment & $73.7(11.1)$ & $74.6(10.7)$ & $-0.9(-6.1,4.3)$ & & & \\
\hline 15 min after treatment & $73.8(12.0)$ & $74.9(12.1)$ & $-1.1(-6.8,4.7)$ & & & \\
\hline \multicolumn{7}{|l|}{ Blood flow velocity (meters/sec) } \\
\hline Initial score & $36.3(11.0)$ & $40.5(10.9)$ & $-3.7(-8.9,1.5)$ & 0.666 & 0.323 & 0.769 \\
\hline After 40 min of treatment & $39.6(10.8)$ & $41.1(11.3)$ & $-1.4(-6.6,3.8)$ & $\left(F_{1,68}=0.19\right)$ & $\left(F_{3,204}=1.17\right)$ & $\left(F_{3,204}=0.38\right)$ \\
\hline 5 min after treatment & $40.6(11.6)$ & $41.6(11.5)$ & $-1.0(-6.4,4.3)$ & & & \\
\hline 10 min after treatment & $40.8(11.6)$ & $40.3(10.5)$ & $-0.2(-5.5,5.2)$ & & & \\
\hline 15 min after treatment & $39.7(10.6)$ & $40.9(10.5)$ & $-1.2(-6.2,3.8)$ & & & \\
\hline
\end{tabular}

Note: scores are presented as the mean (standard deviation) for each variable.

treatment with laser-based devices to treatment by lightemitting diodes in recent years due to the lower cost, lack of coherence, and larger spot size in light-emitting diode devices $[8,47,48]$. Therefore, we used the light-emitting diodes in this study. Color Doppler sonography was used for local blood flow velocity evaluation, and it is widely used in clinical medicine because it is a rapid, simple, accurate, and noninvasive method of objectively monitoring blood flow $[49,50]$. However, this study did not demonstrate an increase in the local arterial blood flow velocity after $40 \mathrm{~min}$ of monochromatic IR therapy over the knee joints in patients with knee OA.

Measuring blood pressure with a conventional manual sphygmomanometer used by a physician in routine clinical practice often reported inconsistent and imprecise blood pressure readings due to patient-physician interaction, failure to minimize patient anxiety, or poor measurement techniques [51]. The "white coat" bias has been demonstrated to be $15 \%$ to $20 \%$ in patients with hypertension [52]. Therefore, blood pressure measurement taken outside the clinic using ambulatory blood pressure monitoring is the gold standard measure [53]. However, due to cost and convenience considerations, automatic devices are commonly used in clinics for blood pressure measurements.

Measuring blood pressure using an automatic device in a clinic and leaving the patient alone in a quiet room for at least 14 minutes rest before measurement were found to minimize the white coat effect and yielded values that were comparable to the ambulatory blood pressure measurements [54-56]. The most innovative features to measure blood pressure by automatic devices were that the cuff must be wrapped snugly around the arm, and the patient must keep proper posture during measurement [57]. Therefore, in this study, to avoid the resting effect on heart rate and blood pressure (as the subjects were lying down for up to $60 \mathrm{~min}$ for treatment and followup) and the white coat effect, all 


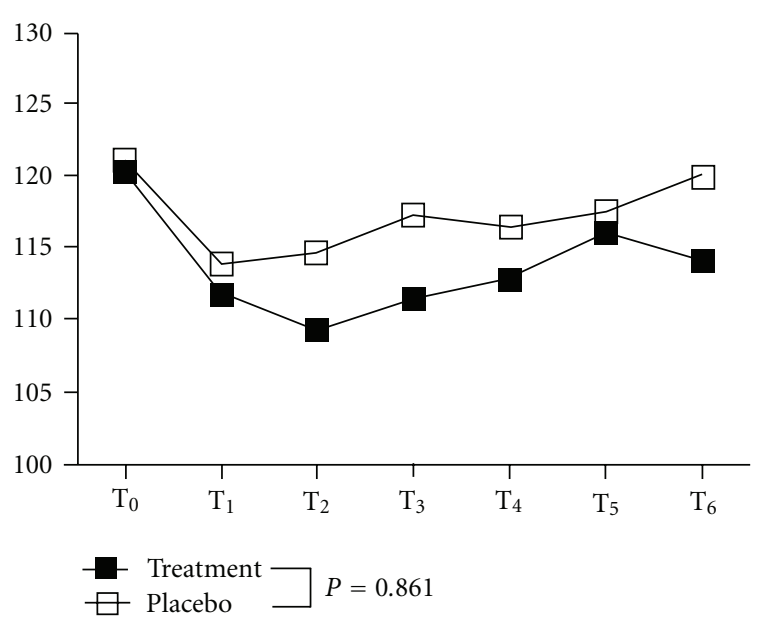

(a)

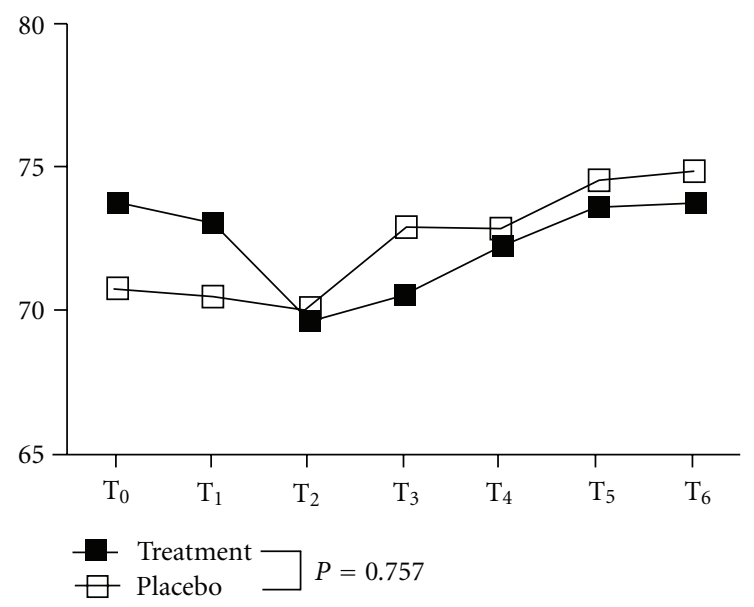

(b)

FIGURE 4: Changes in blood pressure with monochromatic infrared treatment. Solid square: treatment group; hollow square: placebo group; $\mathrm{T}_{0}$ : before treatment; $\mathrm{T}_{1}$ : after $10 \mathrm{~min}$ of treatment; $\mathrm{T}_{2}$ : after 20 min of treatment; $\mathrm{T}_{3}$ : after $30 \mathrm{~min}$ of treatment; $\mathrm{T}_{4}$ : after $40 \mathrm{~min}$ of treatment; $\mathrm{T}_{5}: 5 \mathrm{~min}$ after treatment; $\mathrm{T}_{6}: 15 \mathrm{~min}$ after treatment. (a) systolic blood pressure; (b) diastolic blood pressure. Between groups by repeated-measure ANCOVA: systolic blood pressure: group effect: $P=0.281\left(F_{1,68}=1.18\right)$; time effect: $P=0.180\left(F_{5,335}\right.$ $=1.53)$; group $\mathrm{x}$ time interaction effect: $P=0.861\left(F_{5,335}=0.38\right)$; diastolic blood pressure: group effect: $P=0.262\left(F_{1,68}=0.19\right)$; time effect: $P=0.663\left(F_{5,335}=0.65\right)$; group $\mathrm{x}$ time interaction effect: $P=0.757\left(F_{5,335}=0.53\right)$.

participants were asked to lie on the bed in a quiet room for $15 \mathrm{~min}$ before beginning the blood pressure measurement and intervention. The blood pressure measuring point was $8 \mathrm{~cm}$ above the right elbow joint [57], and all measurements were taken on the right arm. A technician rather than a physician completed the blood pressure measurement. In terms of heart rate and blood pressure, there was no significant difference between the treatment group and placebo group during the $40 \mathrm{~min}$ of treatment or 10 to 15 min after treatment termination.

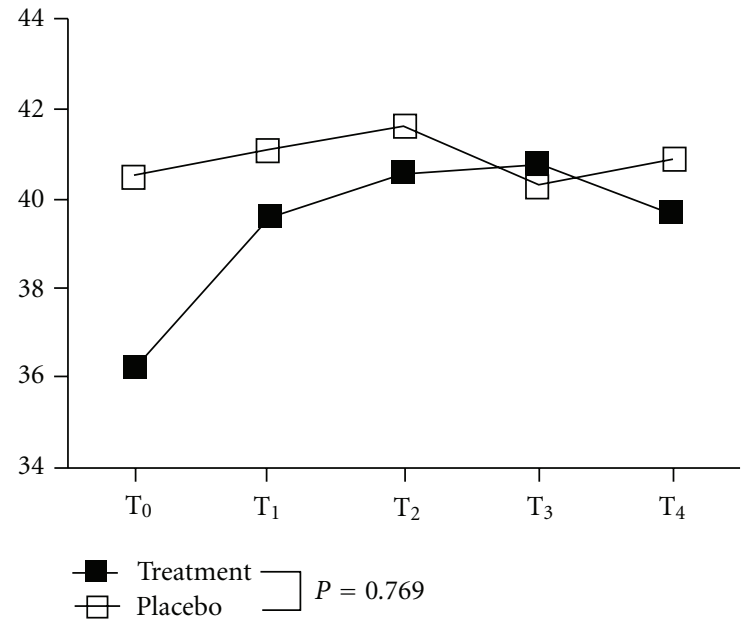

FIGURE 5: Changes in the blood flow velocity with monochromatic radiation treatment. Solid square: treatment group; hollow square: placebo group; $\mathrm{T}_{0}$ : before treatment; $\mathrm{T}_{1}$ : after $40 \mathrm{~min}$ of treatment; $\mathrm{T}_{2}: 5 \mathrm{~min}$ after treatment; $\mathrm{T}_{3}: 10 \mathrm{~min}$ after treatment; $\mathrm{T}_{4}: 15 \mathrm{~min}$ after treatment. Between groups by repeated-measure ANCOVA: group effect: $P=0.666\left(F_{1,68}=0.19\right)$; time effect: $P=0.323\left(F_{3,204}\right.$ $=1.17)$; group $\mathrm{x}$ time interaction effect: $P=0.769\left(F_{3,204}=0.38\right)$.

Compared to a previously conducted, community-based cohort study in Taiwan [58], although the mean age of participants was relatively older, the systolic blood pressure and the diastolic blood pressure mean value ranges were relatively lower in the present study (the respective values for the previously-conducted study in contrast to the present study are as follows: mean age (years): 56.3 in contrast to 61.2 ; mean heart rate (beats/min): 64.8 in contrast to 67.1 ; mean systolic blood pressure (mmHg): 120.2 in contrast to 115.4; mean diastolic blood pressure $(\mathrm{mmHg})$ : 74.1 in contrast to 72.4). In contrast to participants' blood pressures being measured by a conventionl, manual sphygmomanometer in a clinic by a physician without mentioning full rest as in the previous study, the participants' blood pressure monitors were applied by a technician, blood pressure was measured automatically in a quiet room after resting for 15 minutes, and conversation between the technician and participants during heart rate and blood pressure measurements was prohibited in the current study. All of these procedures would effectively minimize the white coat effect and measure blood pressure more accurately. This could partially explain why the ranges of systolic and diastolic blood pressure are lower than the average ranges for 56 years old.

Although all participants were told that they may or may not feel anything from monochromatic IR therapy, active monochromatic IR therapy actually emits mild, tangible heat. Therefore, subjects would expect a difference in perception from monochromatic IR therapy between the 2 groups, which could have given subjects in the treatment group a greater perception that they were being treated in contrast to those in the control group. The percentage of patients who perceived heat in the control group in contrast to the experimental group was $33 \%$ versus $58 \%(P=0.101)$. 
Stratified analysis according to whether the participants perceived heat feeling or not was further performed to control for this factor. For the participants who perceived a heat feeling, we found statistically significant group $\mathrm{x}$ time interactions for heart rate, with higher trends in the control group $(P=0.033)$, and no statistically significant group effect $(P=0.762)$ or time effect $(P=0.708)$. There was no statistically significant group $\mathrm{x}$ time interaction effect for systolic blood pressure $(P=0.543)$, diastolic blood pressure $(P=0.940)$, or knee arterial blood flow velocity $(P=0.323)$. For participants who did not perceive a heat feeling, there was no significant group $\mathrm{x}$ time interaction for heart rate $(P=0.523)$, systolic blood pressure $(P=0.779)$, diastolic blood pressure $(P=0.574)$, or knee arterial blood flow velocity $(P=0.444)$. Although the perceived heat feeling by active monochromatic IR therapy could compromise the experiment's blindness on the patients' side, it does not affect the results after we had controlled for that factor.

Because the effects of monochromatic IR therapy are time-dependent [59], the level of photoenergy delivered would have affected the study's results. Compared to previous studies that applied total energies of $52.0-58.5 \mathrm{~J} / \mathrm{cm}^{2}$ $[11,18,19]$, the present study used $34.7 \mathrm{~mW} / \mathrm{cm}^{2}$ for $40 \mathrm{~min}$ for a higher total energy of $83.2 \mathrm{~J} / \mathrm{cm}^{2}$. At this higher energy, monochromatic IR therapy still had no detrimental systemic cardiovascular effects on patients with knee $\mathrm{OA}$ as measured by the heart rate and blood pressure.

$\mathrm{OA}$ is often associated with obesity and several cardiovascular conditions, including coronary artery disease, hypertension, and diabetes mellitus [60]. Because obesity, hypertension, and cardiovascular diseases are present in metabolic syndrome, it is hypothesized that OA may represent another aspect of metabolic syndrome [61, 62]. Potential mechanisms for joint OA include the following: (1) reduced blood flow from small vessels and interstitial fluid flow in the subchondral bone and (2) subchondral ischemia with compromised gas and nutrient exchange in the articular cartilage [63]. A higher rate of blood flow is associated with an increased bone remodeling rate [63]. On the contrary, compromised blood flow in the subchondral bone could have deleterious effects on the bone and have implications for the cartilage's integrity [63]. There was a positive association between increased popliteal artery vessel wall thickness and generalized OA [62]. These evidences showed that vascular pathology plays a role in joint OA initiation and/or progression [63]. There may be common pathogenic mechanisms that affect the vascular system and joints [61]. Furthermore, most patients with knee OA who require medication for pain relief are likely to be older and at high risk for both adverse cardiovascular and gastrointestinal effects [60]. Therefore, from the point of no detrimental systemic cardiovascular effects, monochromatic IR therapy can be safely applied to elderly people with knee OA and cardiovascular diseases.

Although the present study did not support our previous hypothesis and found no evidence that monochromatic IR therapy increased knee arterial blood flow velocity in patients with knee OA, we acknowledge that there are many factors that could have affected the study results: the photosource, wavelength, power, energy density, duration of treatment, method of application (noncontact mode in contrast to contact mode), site of stimulation, size of the exposure area, and so forth. Therefore, these results cannot be considered conclusive. Our research presents a reasonable initial foray into the local and systemic cardiovascular effects of clinical monochromatic IR therapy application in patients with knee OA.

There are some limitations to the present study. First, no direct NO, prostacyclin or endothelial-derived hyperpolarizing factor productions were measured. Second, whether the increased popliteal blood flow velocity was related to the increased knee joint blood flow and/or arteriole dilatation remains uncertain.

\section{Conclusions}

In this study, we applied monochromatic IR therapy in a double-blind, randomized, and placebo-controlled trial to subjects with knee joint OA. Our results revealed that although there was no increase in knee arterial blood flow velocity, monochromatic IR therapy produced no detrimental systemic cardiovascular effects. Therefore, it can be applied to patients with knee OA and cardiovascular diseases safely. Further studies on the effects of monochromatic IR therapy are warranted in the future using different settings for the power, wavelength, energy density, stimulation duration, and stimulation location.

\section{Acknowledgments}

This study was partially supported by a research grant (SKH8302-98-DR-39) from Shin Kong Wu Ho-Su Memorial Hospital, Taipei, Taiwan, and also partially supported by National Science Council, Taiwan (NSC 99-2628-B-002-061-MY3).

\section{References}

[1] C. Muehleman, A. Margulis, W. C. Bae, and K. Masuda, "Relationship between knee and ankle degeneration in a population of organ donors," BMC Medicine, vol. 8, no. 1, article 48, 2010.

[2] M. C. Hall, S. P. Mockett, and M. Doherty, "Relative impact of radiographic osteoarthritis and pain on quadriceps strength, proprioception, static postural sway and lower limb function," Annals of the Rheumatic Diseases, vol. 65, no. 7, pp. 865-870, 2006.

[3] S. G. Leveille, R. N. Jones, D. K. Kiely et al., "Chronic musculoskeletal pain and the occurrence of falls in an older population," The Journal of the American Medical Association, vol. 302, no. 20, pp. 2214-2221, 2009.

[4] C. B. Swanik, S. M. Lephart, and H. E. Rubash, "Proprioception, kinesthesia, and balance after total knee arthroplasty with cruciate-retaining and posterior stabilized prostheses," Journal of Bone and Joint Surgery Series A, vol. 86, no. 2, pp. 328-334, 2004.

[5] A. Thomas, G. Eichenberger, C. Kempton et al., "Recommendations for the treatment of knee osteoarthritis, using various therapy techniques, based on categorizations of a literature review," Journal of Geriatric Physical Therapy, vol. 32, no. 1, pp. 33-38, 2009. 
[6] R. L. Hsieh and W. C. Lee, "One-shot percutaneous electrical nerve stimulation vs. transcutaneous electrical nerve stimulation for low back pain: comparison of therapeutic effects," American Journal of Physical Medicine and Rehabilitation, vol. 81, no. 11, pp. 838-843, 2002.

[7] F. Fregni, M. Imamura, H. F. Chien et al., "Challenges and recommendations for placebo controls in randomized trials in physical and rehabilitation medicine: a report of the international placebo symposium working group," American Journal of Physical Medicine and Rehabilitation, vol. 89, no. 2, pp. 160$172,2010$.

[8] A. M. Fulop, S. Dhimmer, J. R. Deluca et al., "A meta-analysis of the efficacy of laser phototherapy on pain relief," Clinical Journal of Pain, vol. 26, no. 8, pp. 729-736, 2010.

[9] L. R. Horwitz, T. J. Burke, and D. Carnegie, "Augmentation of wound healing using monochromatic infrared energy. Exploration of a new technology for wound management," Advances in Wound Care, vol. 12, no. 1, pp. 35-40, 1999.

[10] M. W. Powell, D. E. Carnegie, and T. J. Burke, "Reversal of diabetic peripheral neuropathy and new wound incidence: the role of MIRE," Advances in Skin \& Wound Care, vol. 17, no. 6, pp. 295-300, 2004.

[11] D. R. Leonard, M. H. Farooqi, and S. Myers, "Restoration of sensation, reduced pain, and improved balance in subjects with diabetic peripheral neuropathy: a double-blind, randomized, placebo-controlled study with monochromatic nearinfrared treatment," Diabetes Care, vol. 27, no. 1, pp. 168-172, 2004.

[12] A. B. Kochman, D. H. Carnegie, and T. J. Burke, "Symptomatic reversal of peripheral neuropathy in patients with diabetes," Journal of the American Podiatric Medical Association, vol. 92, no. 3, pp. 125-130, 2002.

[13] M. W. Powell, D. H. Carnegie, and T. J. Burke, "Reversal of diabetic peripheral neuropathy with phototherapy (MIRE) decreases falls and the fear of falling and improves activities of daily living in seniors," Age and Ageing, vol. 35, no. 1, pp. 11-16, 2006.

[14] B. Hegedus, L. Viharos, M. Gervain, and M. Galfi, "The effect of low-level laser in knee osteoarthritis: a doubleblind, randomized, placebo-controlled trial," Photomedicine and Laser Surgery, vol. 27, no. 4, pp. 577-584, 2009.

[15] M. Yurtkuran, A. Alp, S. Konur, S. Ozcakir, and U. Bingol, "Laser acupuncture in knee osteoarthritis: a double-blind, randomized controlled study," Photomedicine and Laser Surgery, vol. 25, no. 1, pp. 14-20, 2007.

[16] F. Tascioglu, O. Armagan, Y. Tabak, I. Corapci, and C. Oner, "Low power laser treatment in patients with knee osteoarthritis," Swiss Medical Weekly, vol. 134, no. 17-18, pp. 254-258, 2004.

[17] A. Gur, A. Cosut, A. J. Sarac, R. Cevik, K. Nas, and A. Uyar, "Efficacy of different therapy regimes of low-power laser in painful osteoarthritis of the knee: a double-blind and randomized-controlled trial," Lasers in Surgery and Medicine, vol. 33, no. 5, pp. 330-338, 2003.

[18] J. K. Clifft, R. J. Kasser, T. S. Newton, and A. J. Bush, "The effect of monochromatic infrared energy on sensation in patients with diabetic peripheral neuropathy: a double-blind, placebocontrolled study," Diabetes Care, vol. 28, no. 12, pp. 28962900, 2005.

[19] L. A. Lavery, D. P. Murdoch, J. Williams, and D. C. Lavery, "Does anodyne light therapy improve peripheral neuropathy in diabetes? A double-blind, sham-controlled, randomized trial to evaluate monochromatic infrared photoenergy," Diabetes Care, vol. 31, no. 2, pp. 316-321, 2008.
[20] R. V. da Silva and J. A. Camilli, "Repair of bone defects treated with autogenous bone graft and low-power laser," Journal of Craniofacial Surgery, vol. 17, no. 2, pp. 297-301, 2006.

[21] S. M. J. Abrisham, M. Kermani-Alghoraishi, R. Ghahramani, L. Jabbari, H. Jomeh, and M. Zare, "Additive effects of lowlevel laser therapy with exercise on subacromial syndrome: a randomised, double-blind, controlled trial," Clinical Rheumatology, vol. 30, no. 10, pp. 1341-1346, 2011.

[22] Y. S. Lin, M. H. Huang, C. Y. Chai, and R. C. Yang, "Effects of helium-neon laser on levels of stress protein and arthritic histopathology in experimental osteoarthritis," American Journal of Physical Medicine and Rehabilitation, vol. 83, no. 10, pp. 758-765, 2004.

[23] M. Komori, K. Takada, Y. Tomizawa et al., "Microcirculatory responses to acupuncture stimulation and phototherapy," Anesthesia and Analgesia, vol. 108, no. 2, pp. 635-640, 2009.

[24] Y. Maegawa, T. Itoh, T. Hosokawa, K. Yaegashi, and M. Nishi, "Effects of near-infrared low-level laser irradiation on microcirculation," Lasers in Surgery and Medicine, vol. 27, no. 5, pp. 427-437, 2000.

[25] F. R. M. Ihsan, "Low-level laser therapy accelerates collateral circulation and enhances microcirculation," Photomedicine and Laser Surgery, vol. 23, no. 3, pp. 289-294, 2005.

[26] K. A. Samoilova, N. A. Zhevago, N. N. Petrishchev, and A. A. Zimin, "Role of nitric oxide in the visible light-induced rapid increase of human skin microcirculation at the local and systemic levels: II. Healthy volunteers," Photomedicine and Laser Surgery, vol. 26, no. 5, pp. 443-449, 2008.

[27] B. Braverman, R. J. McCarthy, A. D. Ivankovich, D. E. Forde, M. Overfield, and M. S. Bapna, "Effect of helium-neon and infrared laser irradiation on wound healing in rabbits," Lasers in Surgery and Medicine, vol. 9, no. 1, pp. 50-58, 1989.

[28] N. N. Petrishchev and T. D. Vlasov, Eds., Physiology and Pathophysiology of Endothelium, in: Endothelium Dysfunction: Courses, Mechanisms, Pharmacological Correction, I.P. Pavlov State Medical University, St. Petersburg, Russia, 2003.

[29] K. A. Samoilova, K. D. Obolenskaya, A. V. Vologdina, S. A. Snopov, and E. V. Shevchenko, "Single skin exposure to visible polarized light induces rapid modification of entire circulating blood. 1. Improvement of rheologic and immune parameters," in Proceedings of the Effects of Low-Power Light on Biologigal Systems IV, pp. 90-103, September 1998.

[30] A. Bal, E. Eksioglu, E. Gurcay, B. Gulec, O. Karaahmet, and A. Cakci, "Low-level laser therapy in subacromial impingement syndrome," Photomedicine and Laser Surgery, vol. 27, no. 1, pp. 31-36, 2009.

[31] M. A. Trelles, J. Rigau, R. G. Calderhead et al., "Treatment of knee osteoarthritis with an infrared diode laser," ILTA Okinawa Congress. Laser Therapy, vol. 2, article 26, 1990.

[32] J. Stelian, I. Gil, B. Habot et al., "Improvement of pain and disability in elderly patients with degenerative osteoarthritis of the knee treated with narrow-band light therapy," Journal of the American Geriatrics Society, vol. 40, no. 1, pp. 23-26, 1992.

[33] R. Marks and F. de Palma, "Clinical efficacy of low power laser therapy in osteoarthritis," Physiotherapy Research International, vol. 4, no. 2, pp. 141-157, 1999.

[34] C. Riegger-Krugh and T. Burke, "Outcome in knee osteoarthritis intervention with use of the anodyne system and synvisc," Journal of Orthopaedic \& Sports Physical Therapy, vol. 31, pp. A-38-A-39, 2001.

[35] T. P. Nolan and S. L. Michlovitz, Eds., Monochromatic Infrared Photo Energy (MIRE), F.A. Davis Company, Philadelphia, Pa, USA, 2005. 
[36] R. Altman, E. Asch, D. Bloch et al., "Development of criteria for the classification and reporting of osteoarthritis. Classification of osteoarthritis of the knee. Diagnostic and Therapeutic Criteria Committee of the American Rheumatism Association," Arthritis and Rheumatism, vol. 29, no. 8, pp. 1039-1049, 1986.

[37] N. Bellamy, WOMAC Osteoarthritis User's Guide IX, Victoria Hospital, Ontario, Canada, 2008.

[38] N. Bellamy, "Pain assessment in osteoarthritis: experience with the WOMAC osteoarthritis index," Seminars in Arthritis and Rheumatism, vol. 18, no. 4, pp. 14-17, 1989.

[39] E. M. Roos, M. Klassbo, and L. S. Lohmander, "WOMAC osteoarthritis index. Reliability, validity, and responsiveness in patients with arthroscopically assessed osteoarthritis. Western Ontario and MacMaster Universities," Scandinavian Journal of Rheumatology, vol. 28, no. 4, pp. 210-215, 1999.

[40] N. Bellamy, W. W. Buchanan, C. H. Goldsmith, J. Campbell, and L. W. Stitt, "Validation study of WOMAC: a health status instrument for measuring clinically important patient relevant outcomes to antirheumatic drug therapy in patients with osteoarthritis of the hip or knee," Journal of Rheumatology, vol. 15, no. 12, pp. 1833-1840, 1988.

[41] P. J. Mason, J. E. Manson, H. D. Sesso et al., "Blood pressure and risk of secondary cardiovascular events in women: the women's antioxidant cardiovascular atudy (WACS)," Circulation, vol. 109, no. 13, pp. 1623-1629, 2004.

[42] L. D. Kirwan, N. J. MacLusky, H. M. Shapiro, B. L. Abramson, S. G. Thomas, and J. M. Goodman, "Acute and chronic effects of hormone replacement therapy on the cardiovascular system in healthy postmenopausal women," Journal of Clinical Endocrinology and Metabolism, vol. 89, no. 4, pp. 1618-1629, 2004.

[43] R. L. Hsieh, W. C. Lee, and C. H. Chang, "Maximal cardiovascular fitness and its correlates in ambulatory hemodialysis patients," American Journal of Kidney Diseases, vol. 48, no. 1, pp. 21-27, 2006.

[44] P. Korovessis, P. Iliopoulos, A. Misiris, and G. Koureas, "Color Doppler ultrasonography for evaluation of internal mammary artery application in adolescent female patients with rightconvex thoracic idiopathic scoliosis," Spine, vol. 28, no. 15, pp. 1746-1748, 2003.

[45] A. E. Abbas, F. D. Fortuin, B. Patel, C. A. Moreno, N. B. Schiller, and S. J. Lester, "Noninvasive measurement of systemic vascular resistance using Doppler echocardiography," Journal of the American Society of Echocardiography, vol. 17, no. 8, pp. 834-838, 2004.

[46] A. Schindl, G. Heinze, M. Schindl, H. Pernerstorfer-Schon, and L. Schindl, "Systemic effects of low-intensity laser irradiation on skin microcirculation in patients with diabetic microangiopathy," Microvascular Research, vol. 64, no. 2, pp. 240-246, 2002.

[47] R. L. Hsieh, M. T. Lo, W. C. Liao, and W. C. Lee, "Short-term effects of 890-nanometer radiation on pain, physical activity, and postural stability in patients with knee osteoarthritis: a double-blind, randomized, placebo-controlled study," Archives of Physical Medicine and Rehabilitation, vol. 93, no. 5, pp. 757-764, 2012.

[48] E. C. Leal Junior, V. de Godoi, J. L. Mancalossi et al., "Comparison between cold water immersion therapy (CWIT) and light emitting diode therapy (LEDT) in short-term skeletal muscle recovery after high-intensity exercise in athletes-preliminary results," Lasers in Medical Science, vol. 26, no. 4, pp. 493-501, 2011.
[49] A. N. Khalid, S. A. Quraishi, W. A. Zang, J. L. Chadwick, and B. C. Stack, "Color doppler ultrasonography is a reliable predictor of free tissue transfer outcomes in head and neck reconstruction," Otolaryngology, vol. 134, no. 4, pp. 635-638, 2006.

[50] N. Kalfa, C. Veyrac, M. Lopez et al., "Multicenter assessment of ultrasound of the spermatic cord in children with acute scrotum," Journal of Urology, vol. 177, no. 1, pp. 297-301, 2007.

[51] T. G. Pickering, W. Gerin, J. E. Schwartz, T. M. Spruill, and K. W. Davidson, "Franz Volhard lecture: should doctors still measure blood pressure? The missing patients with masked hypertension," Journal of Hypertension, vol. 26, no. 12, pp. 2259-2267, 2008.

[52] G. Mancia, G. de Backer, A. Dominiczak et al., "ESH/ESC 2007 guidelines for the management of arterial hypertension," Revista Espanola de Cardiologia, vol. 60, no. 9, pp. 968.e1968.e94, 2007.

[53] R. Espinosa, T. M. Spruill, M. J. Zawadzki et al., "Can blood pressure measurements taken in the physician's office avoid the "white coat" bias?" Blood Pressure Monitoring, vol. 16, no. 5, pp. 231-237, 2011.

[54] M. G. Myers, M. Valdivieso, and A. Kiss, "Use of automated office blood pressure measurement to reduce the white coat response," Journal of Hypertension, vol. 27, no. 2, pp. 280-286, 2009.

[55] W. Gerin, R. M. Marion, R. Friedman, G. D. James, D. H. Bovbjerg, and T. G. Pickering, "How should we measure blood pressure in the doctor's office?" Blood Pressure Monitoring, vol. 6, no. 5, pp. 257-262, 2001.

[56] C. Sala, E. Santin, M. Rescaldani, and F. Magrini, "How long shall the patient rest before clinic blood pressure measurement?" American Journal of Hypertension, vol. 19, no. 7, pp. 713-717, 2006.

[57] H. Takahashi, M. Yoshika, and T. Yokoi, "Validation of home blood pressure-monitoring devices, Omron HEM-1020 and Omron i-Q132 (HEM-1010-E), according to the European Society of Hypertension International Protocol," Blood Pressure Monitoring, vol. 16, no. 4, pp. 203-207, 2011.

[58] K. L. Chien, H. W. Chang, Y. T. Lee et al., "Association between physical activities, blood pressure and cholesterol level in Chin-Shan community cardiovascular study, Taiwan," Chinese Journal of Public Health, vol. 18, no. 6, pp. 413-422, 1999.

[59] J. K. Clifft, R. J. Kasser, T. S. Newton, and A. J. Bush, “The effect of monochromatic infrared energy on sensation in subjects with diabetic peripheral neuropathy: a double-blind, placebocontrolled study-response to Burke," Diabetes Care, vol. 29, no. 5, pp. 1186-1187, 2006.

[60] W. B. White, "The potential role of nitric oxide in cardiovascular safety when treating osteoarthritis in patients with hypertension. Introduction," The American Journal of Medicine, vol. 122, no. 5, supplement, pp. S1-S2, 2009.

[61] J. D. Katz, S. Agrawal, and M. Velasquez, "Getting to the heart of the matter: osteoarthritis takes its place as part of the metabolic syndrome," Current Opinion in Rheumatology, vol. 22, no. 5, pp. 512-519, 2010.

[62] P. R. Kornaat, R. Sharma, R. J. van der Geest et al., "Positive association between increased popliteal artery vessel wall thickness and generalized osteoarthritis: is OA also part of the metabolic syndrome?" Skeletal Radiology, vol. 38, no. 12, pp. 1147-1151, 2009.

[63] D. M. Findlay, "Vascular pathology and osteoarthritis," Rheumatology, vol. 46, no. 12, pp. 1763-1768, 2007. 


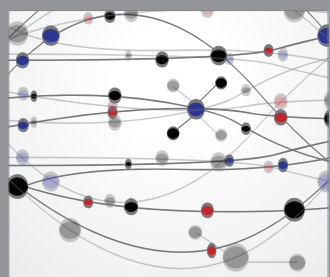

The Scientific World Journal
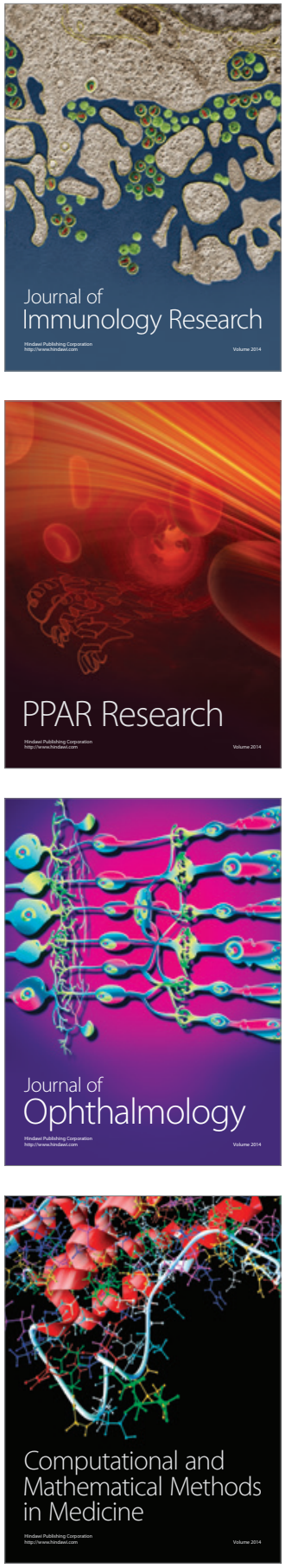

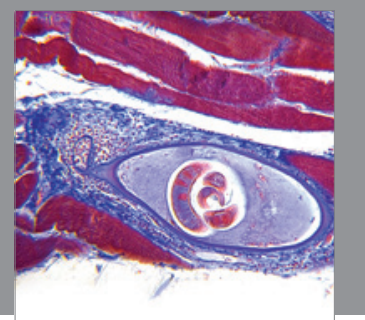

Gastroenterology

Research and Practice
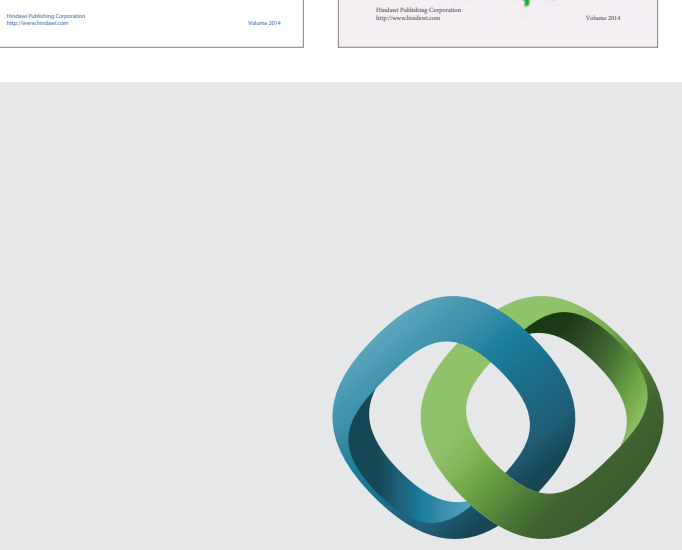

\section{Hindawi}

Submit your manuscripts at

http://www.hindawi.com
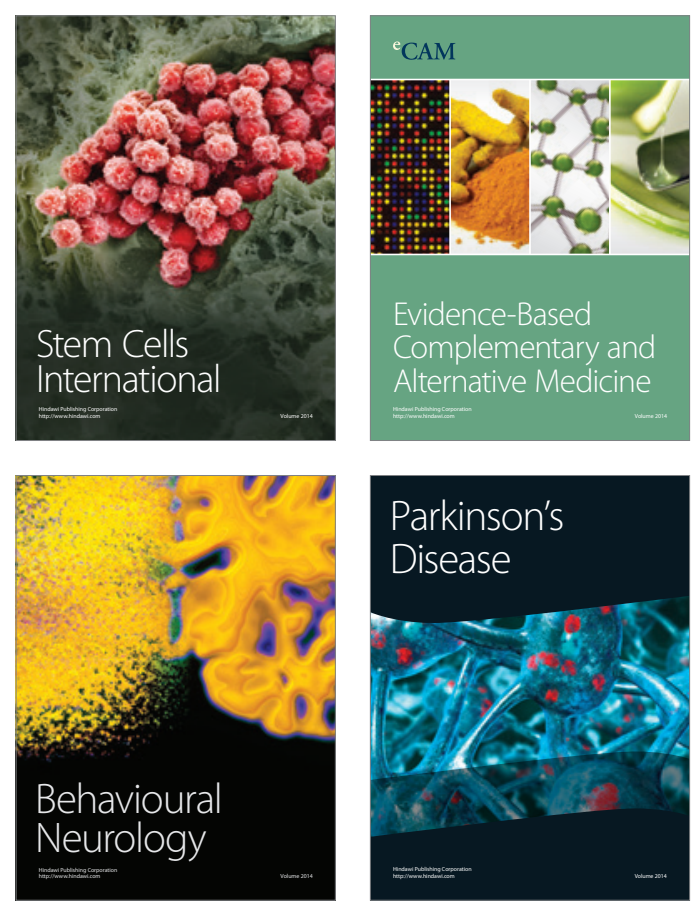

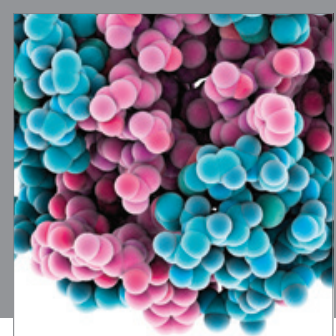

Journal of
Diabetes Research

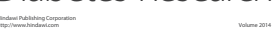

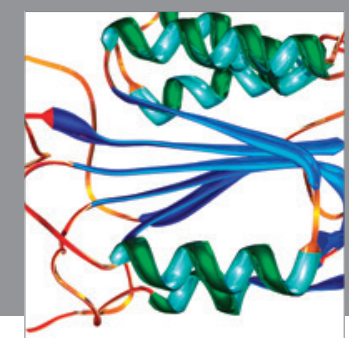

Disease Markers
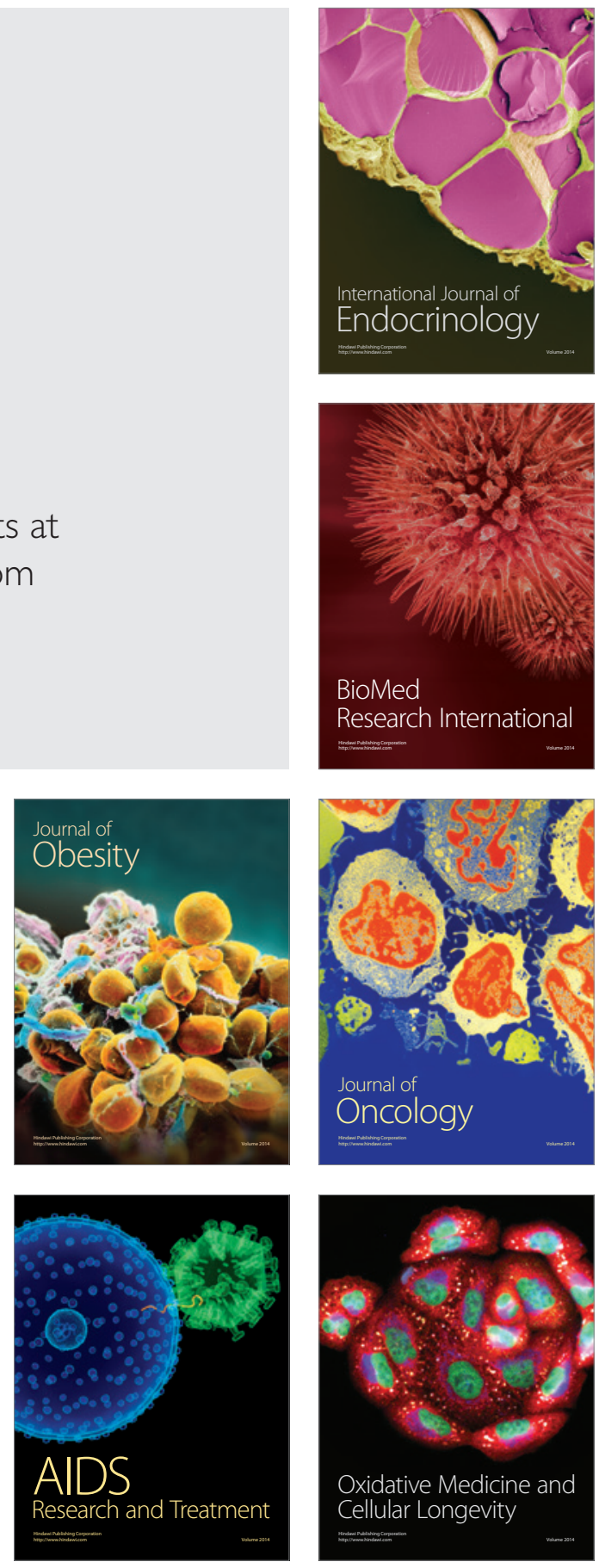